\title{
Receptor for advanced glycation end-products (RAGE) is overexpressed in human osteosarcoma and promotes the proliferation of osteosarcoma $\mathrm{U}-2 \mathrm{OS}$ cells in vitro
}

\author{
Q. Zhang', Y. Jin ${ }^{2}$, C.F. Zhao', W.J. Wang ${ }^{1}$ and G.Y. Liu ${ }^{1}$ \\ ${ }^{1}$ Department of Orthopedics, China-Japan Union Hospital of Jilin University, \\ Changchun, China \\ ${ }^{2}$ Department of Ophthalmology, Second Hospital of Jilin University, \\ Changchun, China \\ Corresponding author: G.Y. Liu \\ E-mail: guangyao1973@126.com
}

Genet. Mol. Res. 15 (2): gmr. 15027817

Received October 9, 2015

Accepted February 12, 2016

Published June 10, 2016

DOI http://dx.doi.org/10.4238/gmr.15027817

\begin{abstract}
Osteosarcoma (OS) is an aggressive cancer of the long bones, and usually affects children and young adults. The receptor for advanced glycation end-products (RAGE) has recently been recognized as an oncogenic receptor that binds to different ligands, and promotes the progression of various cancers. However, little is known about the association between RAGE and the pathogenesis of OS. In this study, we first examined the expression of RAGE in OS tissues using immunohistochemical staining, western blotting, and reverse transcription quantitative polymerase chain reaction. We then determined the influence of the overexpressed RAGE on the proliferation of U-2OS cells in vitro. The results showed that RAGE was overexpressed in OS tissues compared with peritumor tissues, at both the mRNA and protein levels, and there was a significant association between overexpressed RAGE and clinicopathological characteristics, such as clinical stage and
\end{abstract}


distant metastasis. Moreover, the overexpression of RAGE in U-2OS cells significantly promoted their proliferation in vitro. In conclusion, this study indicated that RAGE is overexpressed in OS tissue and promotes the proliferation of U-2OS cells. These data imply that RAGE promotes the growth of OS, and is a potential diagnostic biomarker and therapeutic target for the disorder.

Key words: RAGE; Osteosarcoma; Proliferation; U-2OS cells

\section{INTRODUCTION}

Osteosarcoma (OS) is an aggressive cancer originating in the long bones. It usually affects children and young adults, and metastasizes to the lungs (Ta et al., 2009; Gill et al., 2013). The approximate incidence is 2-3 cases per million per year, and this figure is much higher in young adults and adolescents (Lee, 2015). Depending on the advanced surgical and synthetic therapeutic strategies used, the survival rate of OS patients can increase significantly to $60-70 \%$ (Zhu et al., 2013). In recent years, research has focused on the molecular mechanisms underlying OS in an attempt to find molecular solutions for OS therapy and prognosis. Growth factors, such as transforming growth factor (TGF), connective tissue growth factor (CTGF), and insulin-like growth factor (IGF), are associated with the pathogenesis of OS. These factors could accelerate the proliferation of OS cells and induce oncogenesis. There are also other important aspects of OS pathogenesis such as tumor angiogenesis, transcription factors, and cell adhesion and migration (Broadhead et al., 2011).

Receptor for advanced glycation end-products (RAGE) is an oncogenic receptor that can bind to different ligands (Lee and Park, 2013). The interaction between RAGE and ligands plays an important role in various cellular processes, such as proliferation, apoptosis, autophagy, migration, and inflammation (Lin, 2006; Sims et al., 2010). RAGE is overexpressed in numerous types of cancers, such as gastric (Wang et al., 2015), oral (Su et al., 2015), pancreatic (Kang et al., 2014), colorectal (Dahlmann et al., 2014), and prostate (Zhao et al., 2014) cancers; inactivation of RAGE could inhibit the proliferation and invasion of cancer cells. Therefore, RAGE is a potential diagnostic biomarker and therapeutic target for OS. The regulation of RAGE is important in both disease pathogenesis and cellular homeostasis (Hudson et al., 2008). However, the expression of RAGE in OS tissues is not well understood, and little is known about the association between RAGE and the pathogenesis of OS.

In this study, we examined the expression of RAGE in OS and peritumor tissues by using immunohistochemical staining, and evaluated the expression level of RAGE relative to $\beta$-actin in those tissues. We then determined whether the overexpression of RAGE promotes the proliferation of U-2OS cells in vitro. The results of this study imply that RAGE is overexpressed in human OS and promotes the proliferation of OS U-2OS cells in vitro.

\section{MATERIAL AND METHODS}

\section{OS tissues, cell culture, and transfection}

In this study, OS tissue and peritumor tissue ( $1 \mathrm{~cm}$ away from the OS tissue) was collected by surgical resection from 65 OS patients, who were registered in the Department 
of Orthopedics, China-Japan Union Hospital of Jilin University, between January 2013 and December 2014. OS patients were only selected if it was the first time they had received a diagnosis of OS or if they had not received any treatment prior to commencement of the study. All tissue samples were frozen at $-80^{\circ} \mathrm{C}$ immediately following surgical resection before use. The detailed characteristics of the patients are given in Table 1. Each patient was informed before the surgical operation, and signed a consent form for the use of the excised cancer tissue in medical or scientific research.

Table 1. Association between receptor for advanced glycation end-products (RAGE) overexpression and clinicopathological characteristics of the osteosarcoma patients.

\begin{tabular}{|c|c|c|c|c|}
\hline \multirow[t]{2}{*}{ Characteristic } & \multirow[t]{2}{*}{ Patients (N) } & \multicolumn{2}{|c|}{ RAGE expression } & \multirow[t]{2}{*}{$\mathrm{P}$ value } \\
\hline & & Positive (\%) & Negative (\%) & \\
\hline \multicolumn{5}{|l|}{ Gender } \\
\hline Male & 35 & $24(52.17)$ & $11(57.89)$ & 0.6739 \\
\hline Female & 30 & $22(47.83)$ & $8(42.11)$ & \\
\hline \multicolumn{5}{|l|}{ Age (years) } \\
\hline$\leq 20$ & 43 & $29(63.04)$ & $14(73.68)$ & 0.4096 \\
\hline$>20$ & 22 & $17(36.96)$ & $5(26.32)$ & \\
\hline \multicolumn{5}{|l|}{ Size $(\mathrm{cm})$} \\
\hline$\leq 5.0$ & 32 & $21(45.65)$ & $11(57.89)$ & 0.3692 \\
\hline$>5.0$ & 33 & $25(54.35)$ & $8(42.11)$ & \\
\hline \multicolumn{5}{|l|}{ Clinical stage } \\
\hline $\mathrm{I}+\mathrm{II}$ & 34 & $20(43.48)$ & $14(73.68)$ & 0.0266 \\
\hline III & 31 & $26(56.52)$ & $5(26.32)$ & \\
\hline \multicolumn{5}{|c|}{ Distant metastasis } \\
\hline Yes & 21 & $19(41.30)$ & $2(10.53)$ & 0.0158 \\
\hline No & 44 & $27(58.70)$ & $17(89.47)$ & \\
\hline
\end{tabular}

The U-2OS cells were purchased from the American Type Culture Collection (ATCC) (Rockville, MD, USA). They were cultured in Roswell Park Memorial Institute 1640 (RPMI1640) medium (Ameresco, Framingham, MA, USA) with $10 \%$ fetal bovine serum (FBS) (Invitrogen), $100 \mathrm{U} / \mathrm{mL}$ penicillin, and $100 \mathrm{mg} / \mathrm{mL}$ streptomycin, and incubated at $37^{\circ} \mathrm{C}$ in $5 \%$ $\mathrm{CO}_{2}$. To overexpress RAGE in the U-2OS cells, the RAGE coding sequence was obtained by polymerase chain reaction (PCR) using Phusion polymerase (New England BioLabs, Ipswich, MA, USA), and was cloned into the pcDNA3.1(+) plasmid (Invitrogen). The sequence of the recombinant RAGE-pcDNA3.1(+) plasmid was confirmed by sequencing. U-2OS cells with more than $85 \%$ confluence on a 24 -well plate were transfected with the recombinant RAGEpcDNA3.1(+) plasmid using Lipofectamine ${ }^{\circledR} 2000$ Transfection Reagent (Invitrogen).

\section{Immunohistochemical staining of RAGE}

Immunohistochemical staining was performed for the detection of RAGE at the protein level in the OS tissues. First, the OS and peritumor (control) tissues were fixed in formalin, embedded in paraffin wax $(3 \mathrm{~mm})$, kept at $56^{\circ} \mathrm{C}$ for $1 \mathrm{~h}$, and placed in xylene for 20 min to remove the paraffin. Second, the tissues were subjected to an ethanol gradient (100, $95,90,80$, and $70 \% ; 2$ min for each stage) at $25^{\circ} \mathrm{C}$, and were then treated with a solution of peroxidase-blocking reagent to inactivate the endogenous peroxidase activity. Third, the slides were washed three times with phosphate-buffered saline (PBS) for $10 \mathrm{~min}$, permeabilized with $0.1 \%$ TritonX-100 in PBS for 20 min to break the cytomembrane, and blocked in $1 \%$ bovine serum albumin in PBS for another $30 \mathrm{~min}$ at $37^{\circ} \mathrm{C}$. For the specific binding of RAGE 
to antibody, slides were incubated overnight at $4^{\circ} \mathrm{C}$ with the primary RAGE antibody (mouse monoclonal antibody, Abcam, Cambridge, UK, 1:1000), and then washed three times with PBS. Then slides were incubated with peroxidase anti-mouse $\mathrm{IgG}$ for $30 \mathrm{~min}$ at $37^{\circ} \mathrm{C}$, and finally visualized using 3,3'-diaminobenzidine tetrahydrochloride substrate (Sigma-Aldrich, St. Louis, MO, USA).

\section{Analysis of RAGE using quantitative reverse transcription polymerase chain reaction ( $R T-q P C R)$}

We used RT-qPCR to investigate the expression of RAGE at the mRNA level. The mRNA samples were isolated from the OS tissues or from U-2OS cells lysed with Trizol reagent (Life Technologies, Grand Island, NY, USA) according to the manufacturer instructions. They were then dissolved in RNase-free water and stored at $-70^{\circ} \mathrm{C}$ before use. The RT-qPCR procedure was performed using a SYBR green One-Step RT-PCR kit (TaKaRa, Tokyo, Japan) for each sample according to the manufacturer instructions. After the reaction, the RAGE mRNA level was calculated and was presented as the level of RAGE relative to $\beta$-actin (as control) using the $\Delta \Delta \mathrm{Ct}$ method.

\section{Western blotting analysis}

After harvesting, the U2-OS cells were homogenized in an ice-cold cell lysis buffer (Bio-Rad, Hercules, CA, USA). The cellular lysates were centrifuged at 12,000 $\mathrm{g}$ for $30 \mathrm{~min}$ at $4{ }^{\circ} \mathrm{C}$, and the supernatant was collected. Protein samples were separated by electrophoresis using a $10 \%$ sodium dodecyl sulfate polyacrylamide gel, and were transferred to a nitrocellulose membrane (Millipore, Bedford, MA, USA). After blocking with 5\% skimmed milk powder overnight at $4^{\circ} \mathrm{C}$, the membrane was incubated with the RAGE antibody (mouse monoclonal antibody, Abcam, Cambridge, United Kingdom, 1:500) overnight at $4^{\circ} \mathrm{C}$, and then incubated with horseradish peroxidase (HRP)-linked secondary anti-rabbit antibody (New England BioLabs, Ipswich, United Kingdom) for $1 \mathrm{~h}$ at room temperature. The specific binding band was scanned and quantified according to the band density using the Image $\mathbf{J}$ software.

\section{Statistical analysis}

Statistical analysis was performed using GraphPad Prism software (GraphPad Software, La Jolla, CA, USA). The differences between the RAGE mRNA levels in the OS tissues and the peritumor tissues were analyzed by the Student's $t$-test and one way analysis of variance (ANOVA). Results were considered statistically significant for $\mathrm{P} \leq 0.05$.

\section{RESULTS}

\section{Association between RAGE overexpression and clinicopathological characteristics}

To investigate the overexpression of RAGE in OS patients, we collected 65 OS patients' tissues, and investigated the association between RAGE overexpression and different clinicopathological characteristics: gender, age, tumor size, clinical stage, and distant metastasis. The detailed data are shown in Table 1, and indicate that there were no 
significant differences in RAGE overexpression in gender, age, or tumor size. However, a higher proportion of patients at stage III overexpressed RAGE than patients at stage I+II $(\mathrm{P}=$ $0.0266)$. In addition, there was a significant difference in RAGE expression between patients with and without distant metastasis $(\mathrm{P}=0.0158)$. Therefore, we can conclude that there are associations between RAGE overexpression and clinical stage and distant metastasis.

\section{Immunohistochemical staining in human OS tissues}

To investigate RAGE expression in OS patients, we used immunohistochemical staining in human OS tissues $(\mathrm{N}=65)$ and peritumor tissues as controls $(\mathrm{N}=65)$. The results are shown in Figure 1. The representative immunohistochemical staining of RAGE in human OS tissues (Figure 1B) was completely different from that in the peritumor tissues (Figure 1A). The arrows in Figure 1Bindicate the RAGE in OS tissues after immunohistochemical staining, but there is no similar staining in Figure 1A.The total results for both types of tissue are given in Figure 1C. Of the 65 OS tissues investigated, 46 tested positive for RAGE. However, of the 65 peritumor tissues tested, only 29 were positive for RAGE $(\mathrm{P}=0.0025)$. Thus, there was a significant difference between RAGE expression in OS and peritumor tissues, and RAGE overexpression happened more frequently in OS tissues than in peritumor tissues.

A

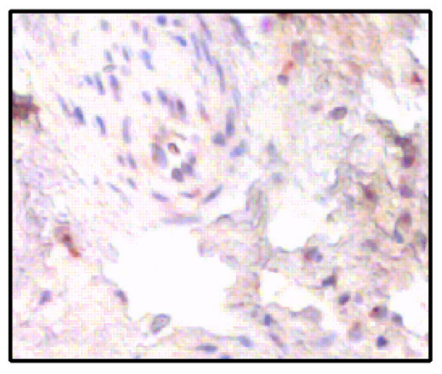

C
B

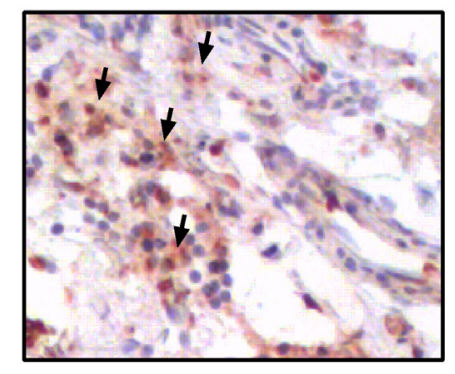

\begin{tabular}{l|c|c|c|c}
\hline \multirow{2}{*}{ Tissues } & Total & \multicolumn{2}{c}{ RAGE expression } & \multirow{2}{*}{ P value } \\
\hline Osteosarcoma & 65 & 46 & 19 & \multirow{2}{*}{0.0025} \\
\hline Peritumor & 65 & 29 & 36 & \\
\hline
\end{tabular}

Figure 1. Immunohistochemical staining in human osteosarcoma tissues. A. and B. Representative immunohistochemical staining for receptor for advanced glycation end-products (RAGE) in human osteosarcoma tissues $(\mathrm{N}=65)$, with peritumor tissues $(\mathrm{N}=65)$ as control $(A)$.

\section{Level of RAGE overexpression in human OS tissues}

To further investigate RAGE expression in OS and peritumor tissues at the mRNA and protein levels, we performed RT-qPCR and western blotting, respectively. As shown in Figure $2 \mathrm{~A}$, the relative mRNA levels of RAGE in OS tissues were much higher than in peritumor 
tissues $(\mathrm{N}=27 ; \mathrm{P}<0.0001)$. The three representative results given in Figure 2Band Figure $2 \mathrm{C}$ show that the protein levels of RAGE in the OS tissues were significantly higher than in the peritumor tissues $(\mathrm{N}=27 ; \mathrm{P}<0.001)$. Therefore, the mRNA and protein levels of RAGE were higher in OS tissues than in peritumor tissues.
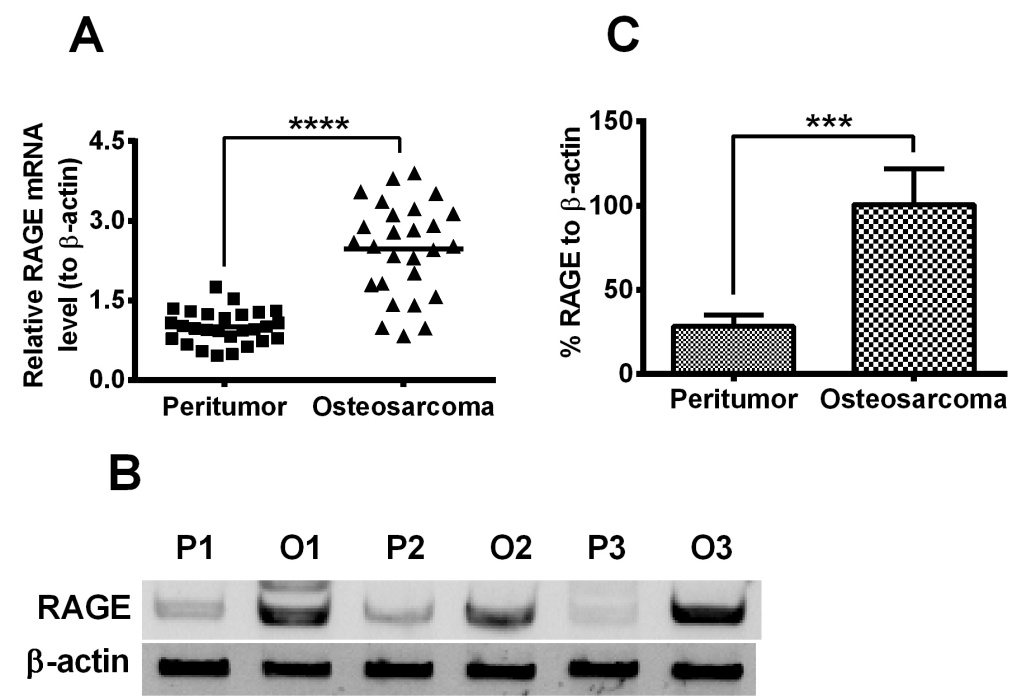

Figure 2. Overexpression of receptor for advanced glycation end-products (RAGE) in human osteosarcoma tissues. A. Relative mRNA level of RAGE in the human osteosarcoma tissues $(\mathrm{N}=27)$. B. Results of western blotting analysis of RAGE and the internal control $(\beta$-actin) in osteosarcoma tissues $(\mathrm{N}=27)$. C. RAGE level presented as a percentage of the $\beta$-actin level. $* * * \mathrm{P}<0.001, * * * * \mathrm{P}<0.0001$.

\section{Overexpressed RAGE promotes the proliferation of U-2OS cells in vitro}

To further investigate the effects of RAGE overexpression in the tumorigenesis of OS, we then overexpressed RAGE using a pcDNA3.1(+) plasmid in OS U-2OS cells, and examined its influence on the growth of U-2OS cells. Normal U-2OS cells and U-2OS cells transfected with Con-pcDNA3.1(+) were used for comparison. Figure 3A indicates that the RAGE mRNA level was almost the same in the normal U-2OS cells and the ConpcDNA3.1(+) plasmid-transfected U-2OS cells. However, the mRNA level in the RAGEpcDNA3.1(+) plasmid-transfected U-2OS cells was much higher than in the other two groups $(\mathrm{P}<0.001)$. The results of western blotting also showed that the expression of RAGE protein was significantly higher in the RAGE-pcDNA3.1(+) plasmid-transfected U-2OS cells than in the normal or Con-pcDNA3.1(+) plasmid-transfected U-2OS cells, using $\beta$-actin as an internal control (Figure 3B; $\mathrm{P}<0.001$ ). Moreover, we determined the survival rates inRAGE-pcDNA3.1(+) plasmid- and Con-pcDNA3.1(+) plasmid-transfected U-2OS cells. Figure $3 \mathrm{C}$ demonstrates that there was a significant difference in proliferation between the RAGE-pcDNA3.1(+) plasmid- and Con-pcDNA3.1(+) plasmid-transfected U-2OS cells at 48 or $72 \mathrm{~h}$ post transfection $(\mathrm{P}<0.01)$. Thus, we confirmed the promotion of $\mathrm{U}-2 \mathrm{OS}$ cell proliferation by RAGE overexpression. 

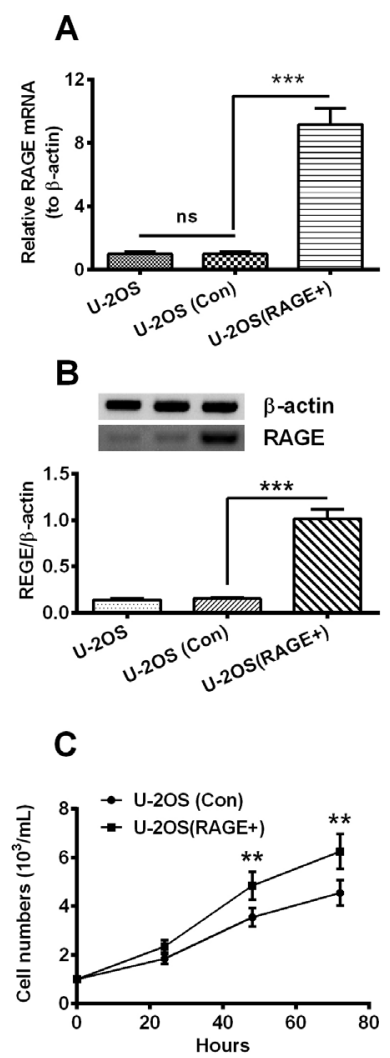

Figure 3. Overexpressed receptor for advanced glycation end-products (RAGE) promotes the proliferation of U-O2S cells in vitro. A. and B. Relative mRNA (A) and protein (B) levels of RAGE in U-O2S cells with or without RAGE overexpression. The RAGE coding sequence was cloned into the pcDNA3.1(+) vector, and the recombinant RAGE-pcDNA3.1(+) or Con-pcDNA3.1(+) plasmid was transfected into U-2OS cells; C. Significantly higher proliferation of U-2OS (RAGE+) cells, the proliferation of U-2OS (Con) or U-2OS (RAGE) was assayed by a counting assay. Data are reported as means $\pm \mathrm{SE}$ of three independent experiments. ${ }^{* *} \mathrm{P}<0.01,{ }^{* * *} \mathrm{P}<0.001$.

\section{DISCUSSION}

Upregulation of RAGE is implicated in many pathological states including cancer, vascular disease, and diabetes (Hudson et al., 2008), and upregulation of RAGE has been shown in various cancers. We investigated the relationship between RAGE expression and clinicopathological characteristics in OS patients, and discovered that the patients with higher clinical stage tended to show overexpression of RAGE. Moreover, high RAGE expression was more prevalent in patients with distant metastasis (Nicolls and Laubach, 2014) than in those without distant metastasis. Immunohistochemical staining revealed the exact location of RAGE in the OS tissues, and the staining results were quite different between the OS and peritumor tissues. This indicated that OS tissues are usually characterized by overexpression of RAGE.

In view of the upregulation of RAGE (Fujita et al., 2007), researchers have proposed its potential use as a diagnostic biomarker and therapeutic target (Lee and Park, 2013). 
However, until now, the expression levels of RAGE in OS tissues have not been reported, and the function of RAGE in OS cells is still unclear. Therefore, we examined RAGE mRNA levels in OS tissues and found them to be much higher than in peritumor tissues. Protein levels of RAGE were also upregulated in OS tissues. All the experiments demonstrated that RAGE was upregulated in OS tissues, suggesting that RAGE contributes to the oncogenesis of OS.

The interaction between RAGE and its ligands plays an important role in critical cellular processes, such as proliferation, apoptosis, and migration (Tesarova et al., 2015). In gastric cancer, knockdown of RAGE reduced the proliferation and invasion of cells, implying that RAGE promotes the proliferation and invasion of cancer cells (Xu et al., 2013; Wang et al., 2015). In this study, we constructed OS U-2OS cells that overexpressed RAGE by transfecting a recombinant plasmid into the cells, and determined the function of RAGE in OS cells. The growth curve of each kind of cell demonstrated a significant difference in proliferation efficiency between the RAGE-overexpressed and control U-2OS cells. Therefore, a high expression level of RAGE promotes the proliferation of U-2OS cells.

In conclusion, this study indicates that RAGE is overexpressed in OS tissues; the expression level of RAGE in OS tissues was much higher than in peritumor tissues. TheRAGEpcDNA3.1(+) plasmid-transfected U-2OS cell model revealed that RAGE promotes the proliferation of cells in vitro. These data imply that RAGE promotes the growth of OS, and is a potential diagnostic biomarker and therapeutic target for the disorder.

\section{Conflicts of interest}

The authors declare no conflict of interest.

\section{ACKNOWLEDGMENTS}

Research supported by the grant from China-Japan Union Hospital of Jilin University.

\section{REFERENCES}

Broadhead ML, Clark JC, Myers DE, Dass CR, et al. (2011). The molecular pathogenesis of osteosarcoma: a review. Sarcoma 2011: 959248. http://dx.doi.org/10.1155/2011/959248

Dahlmann M, Okhrimenko A, Marcinkowski P, Osterland M, et al. (2014). RAGE mediates S100A4-induced cell motility via MAPK/ERK and hypoxia signaling and is a prognostic biomarker for human colorectal cancer metastasis. Oncotarget 5: 3220-3233. http://dx.doi.org/10.18632/oncotarget.1908

Fujita M, Sugama S, Nakai M, Takenouchi T, et al. (2007). alpha-Synuclein stimulates differentiation of osteosarcoma cells: relevance to down-regulation of proteasome activity. J. Biol. Chem. 282: 5736-5748. http://dx.doi.org/10.1074/ jbc.M606175200

Gill J, Ahluwalia MK, Geller D and Gorlick R (2013). New targets and approaches in osteosarcoma. Pharmacol. Ther. 137: 89-99. http://dx.doi.org/10.1016/j.pharmthera.2012.09.003

Hudson BI, Carter AM, Harja E, Kalea AZ, et al. (2008). Identification, classification, and expression of RAGE gene splice variants. FASEB J. 22: 1572-1580. http://dx.doi.org/10.1096/fj.07-9909com

Kang R, Hou W, Zhang Q, Chen R, et al. (2014). RAGE is essential for oncogenic KRAS-mediated hypoxic signaling in pancreatic cancer. Cell Death Dis. 5: e1480. http://dx.doi.org/10.1038/cddis.2014.445

Lee EJ and Park JH (2013). Receptor for advanced glycation end products (RAGE), its ligands, and soluble RAGE: potential biomarkers for diagnosis and therapeutic targets for human renal diseases. Genomics Inform. 11: 224-229. http://dx.doi.org/10.5808/GI.2013.11.4.224

Lee JA (2015). Osteosarcoma in Korean children and adolescents. Korean J. Pediatr. 58: 123-128. http://dx.doi. org/10.3345/kjp.2015.58.4.123 
Lin L (2006). RAGE on the Toll Road? Cell. Mol. Immunol. 3: 351-358.

Nicolls MR and Laubach VE (2014). Traumatic brain injury: lungs in a RAGE. Sci. Transl. Med. 6: 252fs34. http://dx.doi. org/10.1126/scitranslmed.3010259

Sims GP, Rowe DC, Rietdijk ST, Herbst R, et al. (2010). HMGB1 and RAGE in inflammation and cancer. Annu. Rev. Immunol. 28: 367-388. http://dx.doi.org/10.1146/annurev.immunol.021908.132603

Su S, Chien M, Lin C, Chen M, et al. (2015). RAGE gene polymorphism and environmental factor in the risk of oral cancer. J. Dent. Res. 94: 403-411. http://dx.doi.org/10.1177/0022034514566215

Ta HT, Dass CR, Choong PF and Dunstan DE (2009). Osteosarcoma treatment: state of the art. Cancer Metastasis Rev. 28: 247-263. http://dx.doi.org/10.1007/s10555-009-9186-7

Tesarova P, Cabinakova M, Mikulova V, Zima T, et al. (2015). RAGE and its ligands in cancer - culprits, biomarkers, or therapeutic targets? Neoplasma 62: 353-364. http://dx.doi.org/10.4149/neo_2015 061

Wang D, Li T, Ye G, Shen Z, et al. (2015). Overexpression of the Receptor for Advanced Glycation Endproducts (RAGE) is associated with poor prognosis in gastric cancer. PLoS One 10: e0122697. http://dx.doi.org/10.1371/journal. pone. 0122697

Xu XC, Abuduhadeer X, Zhang WB, Li T, et al. (2013). Knockdown of RAGE inhibits growth and invasion of gastric cancer cells. Eur. J. Histochem. 57: e36. http://dx.doi.org/10.4081/ejh.2013.e36

Zhao CB, Bao JM, Lu YJ, Zhao T, et al. (2014). Co-expression of RAGE and HMGB1 is associated with cancer progression and poor patient outcome of prostate cancer. Am. J. Cancer Res. 4: 369-377.

Zhu H, Tang J, Tang M and Cai H (2013). Upregulation of SOX9 in osteosarcoma and its association with tumor progression and patients' prognosis. Diagn. Pathol. 8: 183. http://dx.doi.org/10.1186/1746-1596-8-183 